\title{
Billiard dynamics of bouncing dumbbell
}

\author{
Y. Baryshnikov, V. Blumen, K. Kim, V. Zharnitsky* \\ Department of Mathematics, University of Illinois, Urbana, IL 61801, United States
}

\section{H I G H L I G H T S}

- We study dynamics of a system of two masses connected with a weightless rod, which we call a dumbbell system.

- The dumbbell interacts with a flat boundary.

- We obtain a sharp bound on the number of bounces using billiard techniques.

- In case the ratio of masses is large and the dumbbell rotates fast we find an adiabatic invariant.

\section{A R T I C L E I N F O}

\section{Article history:}

Received 25 June 2013

Received in revised form

9 November 2013

Accepted 12 November 2013

Available online 20 November 2013

Communicated by B. Sandstede

\section{Keywords:}

Bouncing dumbbell

Billiard dynamics

Adiabatic invariant

\begin{abstract}
A B S T R A C T
A system of two masses connected with a weightless rod (called dumbbell in this paper) interacting with a flat boundary is considered. The sharp bound on the number of collisions with the boundary is found using billiard techniques. In case the ratio of masses is large and the dumbbell rotates fast, an adiabatic invariant is obtained.
\end{abstract}

(C) 2013 Elsevier B.V. All rights reserved.

\section{Introduction}

Coin flipping had been already known to ancient Romans as a way to decide an outcome [1]. More recently, scientists inspired by this old question, how unbiased the real (physical) coin is, have been studying coin dynamics, see e.g. [2-4].

Previous studies have mainly focused on the dynamics of the flying coin assuming that it does not bounce and finding the effects of angular momentum on the final orientation. Partial analysis in combination with numerical simulations of the bouncing effects has been done by Vulovic and Prange [5].

On the other hand, there is a well developed theory of mathematical billiards: classical dynamics of a particle moving inside a bounded domain. The particle moves along straight line until it hits the boundary. Next, the particle reflects from the boundary according to Fermat's law. One could expect that the bouncing coin dynamics could be interpreted as a billiard ball problem.

In this paper, we consider a simpler system (with fewer degrees of freedom) which we call the dumbbell. The bouncing coin on a flat surface, restricted to have axis of rotation pointing in the same

\footnotetext{
* Corresponding author. Tel.: +1 2173339576 .

E-mail address: vz@math.uiuc.edu (V.Zharnitsky).
}

direction, can be modeled as a system of two masses connected with a weightless rod.

In the context of statistical mechanics, the billiard ball problem appeared as an attempt to verify physical assumptions about ergodicity of a gas of elastic spheres [6], the so-called Boltzmann ergodic hypothesis.

However, various techniques in billiard dynamics turned out to be useful beyond the original physical problem. The so-called unfolding technique allows one to obtain estimates on the maximal number of bounces of a particle in a wedge [7]. Using this technique, we express the upper bound for the number of bounces of the dumbbell with the flat boundary in terms of the mass ratio. One of the basic questions about the system of hard spheres is to estimate the maximum number of bounces for a system of $N$ hard elastic balls moving freely in an open space [8]. The dumbbell dynamics that is studied in this article can be useful to extend such study to a system of non-spherical particles, see also [9].

Another motivation for the dumbbell dynamics comes from robotics exploratory problems, see e.g. [10]. Consider an automated system that moves in a bounded domain and interacts with the boundary according to some simple laws. In many applications, it is important to cover the whole region as e.g. in automated vacuum cleaners such as Roomba. Then, a natural question arises: what simple mechanical system can generate a dense coverage of a certain subset of the given configuration space? The dumbbell, compared to a 


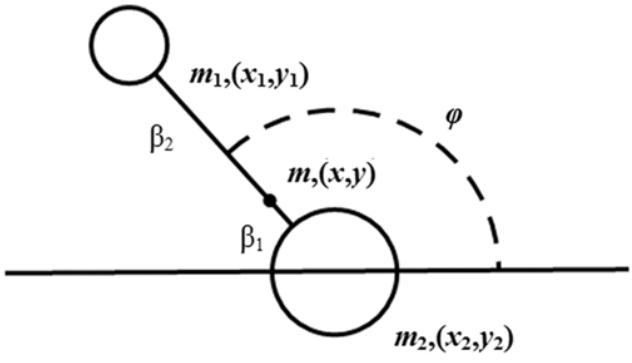

Fig. 1. The system of dumbbell.

material point, has an extra degree of freedom which can generate more chaotic behavior as e.g. in Sinai billiards. Indeed, a rapidly rotating dumbbell will quickly "forget" its initial orientation before the next encounter with the boundary raising some hope for stronger ergodicity.

In this paper, we study the interaction of a dumbbell with the flat boundary. This is an important first step before understanding the full dynamics of the dumbbell in some simple domains. By appropriately rescaling the variables, we obtain an associated single particle billiard problem with the boundary corresponding to the collision curve (which is piecewise smooth) in the configuration space. The number of collisions of the dumbbell with the boundary before scattering out depends on the mass ratio $m_{1} / m_{2}$. If this ratio is far from 1, then the notion of adiabatic invariance can be introduced as there is sufficient time scale separation. We prove an adiabatic invariant type theorem and we describe under what conditions it can be used.

Notation: We use some standard notation when dealing with asymptotic expansions in order to avoid cumbersome use of implicit constants:

$$
\begin{aligned}
& f \lesssim g \Leftrightarrow f=O(g) \Leftrightarrow f \leq C g \text { for some } C>0 \\
& f \gtrsim g \Leftrightarrow g=O(f) \\
& f \approx g \Leftrightarrow f \lesssim g \text { and } f \gtrsim g .
\end{aligned}
$$

\section{Collision laws}

\subsection{Dumbbell-like system}

Let us consider a dumbbell-like system, which consists of two point masses $m_{1}, m_{2}$, connected by a weightless rigid rod of length 1 in the two-dimensional space with coordinates $(x, y)$ without gravity. The coordinates of $m_{1}, m_{2}$, and the center of mass of the system are given by $\left(x_{1}, y_{1}\right),\left(x_{2}, y_{2}\right)$, and $(x, y)$, respectively. Let $\phi$ be the angle measured in the counterclockwise direction from the base line through $m_{1}$ and to the rod. We also define the mass ratios $\beta_{1}=\frac{m_{1}}{m_{1}+m_{2}}$ and $\beta_{2}=\frac{m_{2}}{m_{1}+m_{2}}$ which correspond to the distance from the center of mass to $m_{2}$ and to $m_{1}$, respectively (see Fig. 1).

The dumbbell moves freely in the space until it hits the floor. In this system, the velocity of the center of mass in $x$ direction is constant since there is no force acting on the system in $x$ direction. Thus, we may assume without loss of generality that the center of mass does not move in $x$ direction. With this reduction, the dumbell configuration space is two dimensional with the natural choice of coordinates $(y, \phi)$.

The moment of inertia of the dumbbell is given by $I=m_{1} \beta_{2}^{2}+$ $m_{2} \beta_{1}^{2}=\beta_{1} \beta_{2}\left(m_{1}+m_{2}\right)$. Introducing the total mass $m=m_{1}+m_{2}$, we can write the kinetic energy of the system as

$K=\frac{1}{2} m \dot{y}^{2}+\frac{1}{2} \beta_{1} \beta_{2} m \dot{\phi}^{2}$.

Using the relations

$y_{1}=y+\beta_{2} \sin \phi$ and $y_{2}=y-\beta_{1} \sin \phi$,

we find the velocities of each mass

$\dot{y}_{1}=\dot{y}+\beta_{2} \dot{\phi} \cos \phi$ and $\dot{y}_{2}=\dot{y}-\beta_{1} \dot{\phi} \cos \phi$.

\subsection{Derivation of collision laws}

By rescaling $y=\sqrt{\frac{I}{m}} Y$, we rewrite the kinetic energy

$K=\frac{m}{2} \dot{y}^{2}+\frac{I}{2} \dot{\phi}^{2}=\frac{I}{2}\left(\dot{Y}^{2}+\dot{\phi}^{2}\right)$.

By Hamilton's principle of least action, true orbits extremize

$\int_{t_{0}, Y_{0}, \phi_{0}}^{t_{1}, Y_{1}, \phi_{1}} K(\dot{Y}, \dot{\phi}) \mathrm{d} t$.

Since the kinetic energy is equal to that of the free particle, the trajectories are straight lines between two collisions. When the dumbbell hits the boundary, the collision law is the same as in the classical billiard since in $(Y, \phi)$ coordinates the action is the same. Using the relations

$y_{1}=y+\beta_{2} \sin \phi \geq 0$ and $y_{2}=y-\beta_{1} \sin \phi \geq 0$,

we find the boundaries for the dumbbell dynamics in the $Y-\phi$ plane:

$Y=-\sqrt{\frac{m}{I}} \beta_{2} \sin \phi=-\sqrt{\frac{\beta_{2}}{\beta_{1}}} \sin \phi$

$Y=\sqrt{\frac{m}{I}} \beta_{1} \sin \phi=\sqrt{\frac{\beta_{1}}{\beta_{2}}} \sin \phi$.

The dumbbell hits the floor if one of the above inequalities becomes an equality. Therefore, we take the maximum of two equations to get the boundaries:

$Y=\max \left\{-\sqrt{\beta_{2} / \beta_{1}} \sin \phi, \sqrt{\beta_{1} / \beta_{2}} \sin \phi\right\} \quad$ for $\phi \in[0,2 \pi]$.

Note that this boundary has a non-smooth corner at $\phi=0, \pi$. This is the case when the dumbbell's two masses hit the floor at the same time. We will not consider this degenerate case in our paper.

Now we will derive the collision law for the case when only $m_{1}$ hits the boundary. We recall that given vector $\mathbf{v}_{-}$and a unit vector $\mathbf{n}$ the reflection of $\mathbf{v}_{-}$across $\mathbf{n}$ is given by

$\mathbf{v}_{+}=-2 \frac{\mathbf{v}_{-} \cdot \mathbf{n}}{\mathbf{n} \cdot \mathbf{n}} \mathbf{n}+\mathbf{v}_{-}$.

Here and in the remainder of this paper, $x_{-}, y_{-}, \ldots$ are defined as the corresponding values right before the collision and $x_{+}, y_{+}, \ldots$ are defined as the corresponding values right before the next collision.

According to the collision law, the angle of reflection is equal to the angle of incidence. In our case, $\mathbf{n}$ is the normal vector to the boundary

$Y=-\sqrt{\frac{m}{I}} \beta_{2} \sin \phi$

so that

$\mathbf{n}=\left[1, \sqrt{m / I} \beta_{2} \cos \phi\right]$

$\mathbf{v}_{-}=\left[\dot{Y}_{-}, \dot{\phi}_{-}\right]=\left[\sqrt{m / I} \dot{y}_{-}, \dot{\phi}_{-}\right]$.

Then, using (3), we compute $\mathbf{v}_{+}=\left[\dot{Y}_{+}, \dot{\phi}_{+}\right]$. In this way, we express the translational and the angular velocities after the collision in terms of the velocities before $m_{1}$ hits the floor. Changing back to the original coordinates, we have

$$
\begin{aligned}
\left(\begin{array}{c}
\dot{y}_{+} \\
\dot{\phi}_{+}
\end{array}\right) & =\left(\begin{array}{c}
\sqrt{\frac{I}{m}} \dot{Y}_{+} \\
\dot{\phi}_{+}
\end{array}\right) \\
& =\left(\begin{array}{l}
\dot{y}_{-}\left(-1+\frac{2 \beta_{2} \cos ^{2} \phi}{\beta_{1}+\beta_{2} \cos ^{2} \phi}\right)-\dot{\phi}_{-}\left(\frac{2 \beta_{1} \beta_{2} \cos \phi}{\beta_{1}+\beta_{2} \cos ^{2} \phi}\right) \\
\dot{\phi}_{-}\left(1-\frac{2 \beta_{2} \cos ^{2} \phi}{\beta_{1}+\beta_{2} \cos ^{2} \phi}\right)-\dot{y}_{-}\left(\frac{2 \cos \phi}{\beta_{1}+\beta_{2} \cos ^{2} \phi}\right)
\end{array}\right) .
\end{aligned}
$$




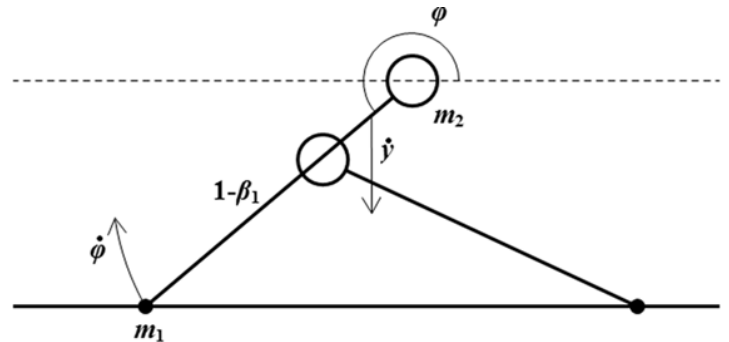

Fig. 2. The light mass bounces many times off the floor while the large mass slowly approaches the floor.

Remark 2.1. The bouncing law for the other case, when $m_{2}$ hits the boundary can be obtained in a similar manner: we switch $\beta_{1}$ and $\beta_{2}$, replace $\cos \phi$ and $\sin \phi$ with $-\cos \phi$ and $-\sin \phi$, and replace $y_{1}$ with $y_{2}$

\section{Adiabatic invariant}

Consider the case when $m_{1} \ll m_{2}$ and $m_{1}$ rotates around $m_{2}$ with high angular velocity $\dot{\phi}$ and assume that the center of mass has slow downward velocity compared to $\phi$. Since multiplying velocities $(\dot{\phi}, \dot{y})$ by a constant does not change the orbit, we normalize $\dot{\phi}$ to be of order 1 , then $\dot{y}$ is small. Consider such dumbell slowly approaching the floor, rotating with angular velocity of order 1, i.e. $\phi \sim 1$.

At some moment the small mass $m_{1}$ will hit the floor. If the angle $\phi=\pi / 2$ (or sufficiently close to it), then the dumbbell will bounce away without experiencing any more collisions. This situation is rather exceptional.

A simple calculation shows that $|\phi-3 \pi / 2|$ will be generically of order $\sqrt{|\dot{y}|}$ for our limit $\dot{y} \rightarrow 0$. In this section we assume this favorable scenario. For the corresponding set of initial conditions, we obtain an adiabatic invariant (nearly conserved quantity). We start by deriving approximate map between two consecutive bounces.

Lemma 3.1. Let $\beta_{1}=\epsilon \ll 1, \dot{\phi}_{-} \neq 0$ and assume $m_{1}$ bounces off the floor and hits the floor next before $m_{2}$ does. Then there exist $\delta=$ $\delta(\epsilon)$ such that if $\delta / \epsilon \rightarrow \infty$ and $\epsilon \rightarrow 0$, and if $-\delta<\dot{y}_{-}<0$ and $\left|\phi-\frac{3 \pi}{2}\right| \gtrsim \sqrt{\delta}$, the collision map is given by

$\dot{\phi}_{+}=-\dot{\phi}_{-}-\frac{2}{\sqrt{1-y_{-}^{2}}} \dot{y}_{-}+O\left(\frac{\epsilon}{\delta}\right)$

$y_{+}=y_{-}-\frac{2 \pi-2 \arccos y_{-}}{\dot{\phi}_{-}} \dot{y}_{-}+O\left(\delta^{3 / 2}\right)+O\left(\frac{\epsilon}{\sqrt{\delta}}\right)$.

Proof. We prove (5) in two steps. We first show that

$\dot{\phi}_{+}=-\dot{\phi}_{-}-\frac{2}{\cos \phi} \dot{y}_{-}+O\left(\frac{\epsilon}{\delta}\right)$

using the expression for $\dot{\phi}_{+}$in (4). We have,

$$
\begin{aligned}
\dot{\phi}_{+} & +\left(\dot{\phi}_{-}+\frac{2}{\cos \phi} \dot{y}_{-}\right)=\left(1-\frac{2\left(1-\beta_{1}\right) \cos ^{2} \phi}{\beta_{1}+\left(1-\beta_{1}\right) \cos ^{2} \phi}\right) \dot{\phi}_{-} \\
& +\left(\frac{2 \cos \phi}{\beta_{1}+\left(1-\beta_{1}\right) \cos ^{2} \phi}\right) \dot{y}_{-}+\left(\dot{\phi}_{-}+\frac{2}{\cos \phi} \dot{y}_{-}\right) \\
= & \frac{\left(\beta_{1}-\left(1-\beta_{1}\right) \cos ^{2} \phi\right) \dot{\phi}_{-}-(2 \cos \phi) \dot{y}_{-}}{\beta_{1} \sin ^{2} \phi+\cos ^{2} \phi} \\
& +\left(\dot{\phi}_{-}+\frac{2}{\cos \phi} \dot{y}_{-}\right) \\
= & \beta_{1}\left(\frac{\dot{\phi}_{-}+1+2 \dot{y}_{-}\left(\frac{\sin ^{2} \phi}{\cos \phi}\right)}{\beta_{1} \sin ^{2} \phi+\cos ^{2} \phi}\right) .
\end{aligned}
$$

For sufficiently small $\delta,\left|\phi-\frac{3 \pi}{2}\right| \gtrsim \sqrt{\delta}$ implies $\cos \phi \gtrsim \sqrt{\delta}$. It follows that

$\left|\dot{\phi}_{+}+\left(\dot{\phi}_{-}+\frac{2}{\cos \phi} \dot{y}_{-}\right)\right| \lesssim \epsilon\left|\frac{1+\frac{2 \delta}{\sqrt{\delta}}}{\delta}\right|=O\left(\frac{\epsilon}{\delta}\right)$.

We observe from Fig. 2 that $\phi=\frac{3 \pi}{2}-\arccos \left(\frac{y_{-}}{1-\beta_{1}}\right)$. Thus,

$$
\begin{aligned}
& \dot{\phi}_{+}+\left(\dot{\phi}_{-}+\frac{2}{\cos \phi} \dot{y}_{-}\right) \\
& =\dot{\phi}_{+}+\dot{\phi}_{-}+\frac{2}{\cos \left(\frac{3 \pi}{2}-\arccos \left(\frac{y_{-}}{1-\beta_{1}}\right)\right)} \dot{y}_{-} \\
& =\dot{\phi}_{+}+\dot{\phi}_{-}+\frac{2}{\sqrt{1-\left(\frac{y_{-}}{1-\beta_{1}}\right)^{2}}} \dot{y}_{-} \\
& =\dot{\phi}_{+}+\dot{\phi}_{-}+\frac{2}{\sqrt{1-y_{-}^{2}}} \dot{y}_{-}+R_{1},
\end{aligned}
$$

where

$\left|R_{1}\right| \leq \beta_{1}\left|\frac{2 \dot{y}_{-} y_{-}}{\sqrt{\left(\left(1-\beta_{1}\right)^{2}-y_{-}^{2}\right)^{3}}}\right|$.

Using that $\sqrt{\left(1-\beta_{1}\right)^{2}-y_{-}^{2}}=\left(1-\beta_{1}\right) \cos \phi$, we obtain

$\left|R_{1}\right| \lesssim \epsilon\left|\frac{2 \delta}{\left(\left(1-\beta_{1}\right) \sqrt{\delta}\right)^{3}}\right|$.

Combining the results, we have

$$
\begin{aligned}
& \left|\dot{\phi}_{+}+\dot{\phi}_{-}+\frac{2}{\sqrt{1-y_{-}^{2}}} \dot{y}_{-}\right|=\left|\dot{\phi}_{+}+\left(\dot{\phi}_{-}+\frac{2}{\cos \phi} \dot{y}_{-}\right)\right|+\left|R_{1}\right| \\
& \lesssim \epsilon\left(\left|\frac{1+\frac{2 \delta}{\sqrt{\delta}}}{\delta}\right|+\left|\frac{2 \delta}{\left(\left(1-\beta_{1}\right)\right)^{3} \delta^{3 / 2}}\right|\right)=O\left(\frac{\epsilon}{\delta}\right) .
\end{aligned}
$$

This completes the proof for (5).

Let $t$ be the time between the two consecutive collisions of $m_{1}$. Then $y_{+}=y_{-}-\dot{y}_{-} t$. The angular distance that $m_{1}$ traveled is given by

$$
\begin{aligned}
\psi & =2 \pi-\arccos \left(\frac{y_{-}}{1-\beta_{1}}\right)-\arccos \left(y_{+}\right) \\
& =2 \pi-\arccos \left(\frac{y_{-}}{1-\beta_{1}}\right)-\arccos \left(\frac{y_{-}-\dot{y}_{-} t}{1-\beta_{1}}\right) \\
& =2 \pi-2 \arccos \left(\frac{y_{-}}{1-\beta_{1}}\right)+R_{2} \\
& =2 \pi-2 \arccos y_{-}+R_{3}+R_{2},
\end{aligned}
$$

where $R_{2}$ and $R_{3}$ are the error estimates for the Taylor series expansion and are given explicitly by

$$
\begin{aligned}
& \left|R_{2}\right| \leq\left|\frac{\dot{y}_{-} t}{\sqrt{\left(1-\beta_{1}\right)^{2}-y_{-}^{2}}}\right| \\
& \left|R_{3}\right| \leq\left|\frac{\beta_{1} y_{-}}{\left(1-\beta_{1}\right) \sqrt{\left(1-\beta_{1}\right)^{2}-y_{-}^{2}}}\right| .
\end{aligned}
$$


Therefore, we have

$$
\begin{aligned}
y_{+} & =y_{-}-\dot{y}_{-} t=y_{-}-\dot{y}_{-}\left(\frac{\psi}{\dot{\phi}_{-}}\right) \\
& =y_{-}-\frac{\dot{y}_{-}}{\dot{\phi}_{-}}\left(2 \pi-2 \arccos y_{-}+R_{2}+R_{3}\right) \\
& =y_{-}-\frac{2 \pi-2 \arccos y_{-}}{\dot{\phi}_{-}} \dot{y}_{-}+\frac{\dot{y}_{-}}{\dot{\phi}_{-}}\left(R_{2}+R_{3}\right) .
\end{aligned}
$$

Since $m_{1}$ can travel at most $2 \pi$ between two collisions, $t$ is bounded by $|t|<\frac{2 \pi}{\dot{\phi}}$. Also note that $R_{2}$ and $R_{3}$ contain the factor $\dot{y}_{-}$and $\beta_{1}$ respectively. We finish the proof for (6) by computing,

$$
\begin{aligned}
& \left|y_{+}-y_{-}+\frac{2 \pi-2 \arccos y_{-}}{\dot{\phi}_{-}} \dot{y}_{-}\right| \lesssim\left|\frac{\dot{y}_{-}}{\dot{\phi}_{-}}\left(R_{2}+R_{3}\right)\right| \\
& \lesssim \dot{y}_{-}^{2}\left|\frac{2 \pi y_{-}}{\dot{\phi}_{-} \sqrt{\left(1-\beta_{1}\right)^{2}-y_{-}^{2}}}\right| \\
& +\beta_{1}\left|\frac{2 \pi y_{-}}{\dot{\phi}_{-}\left(1-\beta_{1}\right) \sqrt{\left(1-\beta_{1}\right)^{2}-y_{-}^{2}}}\right| \\
& \lesssim \delta^{2}\left|\frac{2 \pi}{\sqrt{\delta}}\right|+\epsilon\left|\frac{2 \pi}{\left(\left(1-\beta_{1}\right)\right)^{2} \sqrt{\delta}}\right|=O\left(\delta^{3 / 2}\right)+O\left(\frac{\epsilon}{\sqrt{\delta}}\right) .
\end{aligned}
$$

Corollary 3.2. Under the same assumptions as in Lemma 3.1 with the exception $\left|\phi-\frac{3 \pi}{2}\right| \gtrsim \delta^{k}$ for $0 \leq k \leq 1$ and $\epsilon \ll \delta^{2 k}$, the variables after the collision are given by the similar equations to (5) and (6) but with different error terms.

$$
\begin{aligned}
& \dot{\phi}_{+}=-\dot{\phi}_{-}-\frac{2}{\sqrt{1-y_{-}^{2}}} \dot{y}_{-}+O\left(\frac{\epsilon}{\delta^{2 k}}\right) \\
& y_{+}=y_{-}-\frac{2 \pi-2 \arccos y_{-}}{\dot{\phi}_{-}} \dot{y}_{-}+O\left(\frac{\delta^{2}}{\delta^{k}}\right)+O\left(\frac{\epsilon}{\delta^{k}}\right) .
\end{aligned}
$$

Proof. When computing the error terms, use $\cos \phi \gtrsim \delta^{k}$.

Now, we can state the adiabatic invariance theorem for the special case when the light mass hits the floor and the dumbbell is far away from the vertical position: $\phi=3 \pi / 2$.

Theorem 3.3. Suppose right before the collision $\dot{\phi}_{-} \neq 0$ and $\phi-$ $\frac{3 \pi}{2} \neq 0$. Then there is $\delta>0$ such that if $0<\beta_{1}=\epsilon=\delta^{2}$ and $-\delta<$ $\dot{y}_{0}<0$, then there exists an adiabatic invariant of the dumbbell system, given by $I=|\dot{\phi}| f(y)$, where $f(y)=\pi-\arccos y$. In other words, $\left|\dot{\phi}_{n}\right| f\left(y_{n}\right)-\left|\dot{\phi}_{0}\right| f\left(y_{0}\right)=O(\delta)$ after $N=O\left(\delta^{-1}\right)$ collisions.

Proof. We prove this by finding $f(y)$ that satisfies

$$
\left|\dot{\phi}_{+}\right| f\left(y_{+}\right)-\left|\dot{\phi}_{-}\right| f\left(y_{-}\right)=O\left(\delta^{2}\right) \text {. }
$$

When $\epsilon=\delta^{2}$ and $\delta$ is sufficiently small, it follows from (6a) that,

$f\left(y_{+}\right)=f\left(y_{-}\right)-\left(\frac{2 \pi-2 \arccos y_{-}}{\dot{\phi}_{-}} \dot{y}_{-}+O\left(\delta^{2}\right)\right) f^{\prime}\left(y_{-}\right)$.

Then, we have

$$
\begin{aligned}
& \left|\dot{\phi}_{+}\right| f\left(y_{+}\right)=\left|\dot{\phi}_{-}+\frac{2 \dot{y}_{-}}{\sqrt{1-y_{-}^{2}}}+O\left(\delta^{2}\right)\right| \\
& \quad \times\left(f\left(y_{-}\right)-\left(\frac{2 \pi-2 \arccos y_{-}}{\dot{\phi}_{-}} \dot{y}_{-}+O\left(\delta^{2}\right)\right) f^{\prime}\left(y_{-}\right)\right)
\end{aligned}
$$

$$
\begin{aligned}
& =\left|\dot{\phi}_{-}\right| f\left(y_{-}\right)-\left(2 \pi-2 \arccos y_{-}\right) \dot{y}_{-} f^{\prime}\left(y_{-}\right) \\
& +\left|\frac{2}{\sqrt{1-y_{-}^{2}}} \dot{y}_{-}\right| f\left(y_{-}\right)+O\left(\delta^{2}\right) .
\end{aligned}
$$
vided

Therefore, $f(y)$ satisfies $\left|\dot{\phi}_{+}\right| f\left(y_{+}\right)-\left|\dot{\phi}_{-}\right| f\left(y_{-}\right)=O\left(\delta^{2}\right)$ pro$-\left(2 \pi-2 \arccos y_{-}\right) \dot{y}_{-} f^{\prime}\left(y_{-}\right)+\frac{2}{\sqrt{1-y_{-}^{2}}} \dot{y}_{-} f\left(y_{-}\right)=0$.

The solution of the above equation is given by

$f\left(y_{-}\right)=\pi-\arccos y_{-}$.

Let $N=O\left(\delta^{-1}\right)$ and let $\dot{\phi}_{N}$ and $y_{N}$ be the angular velocity and the distance after $N$ th collision. Then, we have

$$
\begin{aligned}
& \left|\dot{\phi}_{N}\right| f\left(y_{N}\right)-\left|\dot{\phi}_{0}\right| f\left(y_{0}\right) \\
& \quad=\sum_{k=1}^{N}\left(\left|\dot{\phi}_{k}\right| f\left(y_{k}\right)-\left|\dot{\phi}_{k-1}\right| f\left(y_{k-1}\right)\right) \lesssim N \cdot \delta^{2} \lesssim \delta .
\end{aligned}
$$

Remark 3.4. Adiabatic invariant has a natural geometric meaning: angular velocity times the distance traveled by the light mass between two consecutive collisions.

Now, we state the theorem for a realistic scenario when a rapidly rotating dumbell scatters off the floor.

Theorem 3.5. Let the dumbbell approach the floor from infinity with $\dot{\phi}_{-} \neq 0$. There exists $\delta>0$ such that if $0<\beta_{1}=\epsilon=\delta^{2},-\delta<$ $\dot{y}_{-}<0,\left|\phi_{0}-\frac{3 \pi}{2}\right| \sim \sqrt{\delta}$ then, after $N=O\left(\delta^{-1}\right)$ bounces the dumbbell will leave the floor after the final bounce by $m_{1}$ with $I_{N}=I_{0}+$ $O(\sqrt{\delta})$. The adiabatic invariant is defined as above $I=|\dot{\phi}| f(y)$.

Remark 3.6. The condition on the angle $\left|\phi_{0}-\frac{3 \pi}{2}\right| \sim \sqrt{\delta}$ comes naturally from the following argument. If $\dot{y}=-\delta$, the dumbbell approaching from infinity will naturally hit the floor when $y \gtrsim 1-$ $\beta_{1}-\delta$. Since $\delta$ is small, this implies $\left|\phi_{0}-\frac{3 \pi}{2}\right| \lesssim \sqrt{\delta}$. If $\phi_{0}$ happens to be too close to $3 \pi / 2$, then there is no hope to obtain adiabatic invariant and we exclude such set of initial conditions. In the limit $\delta \rightarrow 0$ the relative measure of the set where $\left|\phi_{0}-\frac{3 \pi}{2}\right|=o(\sqrt{\delta})$ tends to zero.

Proof. We will split the iterations (bounces) into two parts: before the $n$th iteration and after it, where $n=[\mu / \sqrt{\delta}]$ and $\mu$ is sufficiently small (to be defined later). We claim that after $n$ bounces, $\left|\phi_{n}-\frac{3 \pi}{2}\right| \gtrsim \sqrt[4]{\delta}$. To prove this claim, we use energy conservation of the dumbbell system (1), and (5a). We have

$$
\begin{aligned}
& \epsilon(1-\epsilon)\left(\dot{\phi}_{-}+\frac{2}{\sqrt{1-y_{-}^{2}}} \dot{y}_{-}+O\left(\frac{\epsilon}{\delta}\right)\right)^{2}+\dot{y}_{+}^{2} \\
& =\epsilon(1-\epsilon) \dot{\phi}_{-}^{2}+\dot{y}_{-}^{2} .
\end{aligned}
$$

Next,

$$
\begin{aligned}
\left|\dot{y}_{+}^{2}-\dot{y}_{-}^{2}\right|=\mid \epsilon(1-\epsilon) \dot{\phi}_{-}^{2}-\epsilon(1-\epsilon) \\
\\
\times\left(\dot{\phi}_{-}+\frac{2}{\sqrt{1-y_{-}^{2}}} \dot{y}_{-}+O\left(\frac{\epsilon}{\delta}\right)\right)^{2} \mid
\end{aligned}
$$




$$
\begin{gathered}
\leq \mid \epsilon\left(\frac{4 \dot{y}_{-}^{2}}{1-y_{-}^{2}}+\frac{4 \dot{\phi}_{-} \dot{y}_{-}}{\sqrt{1-y_{-}^{2}}}+2 \dot{\phi}_{-} O\left(\frac{\epsilon}{\delta}\right)\right. \\
\left.+\frac{4 \dot{y}_{-}}{\sqrt{1-y_{-}^{2}}} O\left(\frac{\epsilon}{\delta}\right)+O\left(\frac{\epsilon}{\delta}\right)^{2}\right) \mid .
\end{gathered}
$$

By our assumptions, $1-y_{-} \gtrsim \delta$ so it follows that

$\left|\dot{y}_{+}^{2}-\dot{y}_{-}^{2}\right| \lesssim \delta^{2}\left(\frac{\delta^{2}}{\delta}+\frac{\delta}{\sqrt{\delta}}+\delta+\frac{\delta^{2}}{\sqrt{\delta}}+\delta^{2}\right) \lesssim \delta^{5 / 2}$,

which implies

$\left|\dot{y}_{+}-\dot{y}_{-}\right| \lesssim \delta^{3 / 2}$.

After $n=\lfloor\mu / \sqrt{\delta}\rfloor$ bounces, $\left|\dot{y}_{n}-\dot{y}_{0}\right| \leq \delta / 2$ if $\mu$ is sufficiently small and we still have the vertical velocity of same order, i.e. $\dot{y}_{n} \sim \dot{y}_{0} \sim \delta$. Then, at the $n$th collision, the center of mass will be located at $y_{n} \lesssim 1-\sqrt{\delta}$, which will imply $\left|\phi_{n}-\frac{3 \pi}{2}\right| \gtrsim \sqrt[4]{\delta}$. Now using Lemma 3.1, Corollary 3.2, and Theorem 3.3, we compute the error term of the adiabatic invariant under the assumption that the total number of collisions is bounded by $N \lesssim \delta^{-1}$ and the heavy mass does not hit the floor.

$$
\begin{aligned}
& \left|\dot{\phi}_{N}\right| f\left(y_{N}\right)-\left|\dot{\phi}_{0}\right| f\left(y_{0}\right)=\sum_{k=1}^{n}\left(\left|\dot{\phi}_{k}\right| f\left(y_{k}\right)-\left|\dot{\phi}_{k-1}\right| f\left(y_{k-1}\right)\right) \\
& \quad+\sum_{n}^{N}\left(\left|\dot{\phi}_{k}\right| f\left(y_{k}\right)-\left|\dot{\phi}_{k-1}\right| f\left(y_{k-1}\right)\right) \\
& =\frac{\mu}{\sqrt{\delta}} O(\delta)+\left(\frac{C}{\delta}\right) O\left(\delta^{3 / 2}\right)=O(\sqrt{\delta}) .
\end{aligned}
$$

By the theorem proved in the next section there is indeed a uniform bound on the number of bounces.

If the heavy mass does hit the floor it can do so only once as shown in the next section. We claim that the corresponding change in the adiabatic invariant will be only of order $\delta$. Indeed, using formula (4) and the comment after that, we obtain

$\dot{y}_{+}=-\dot{y}_{-}+O(\epsilon)$

$\dot{\phi}_{+}=\dot{\phi}_{-}+O(\epsilon)+O(\delta)$,

where subscripts \pm denote the variables just after and before the larger mass hits the floor.

Let the pairs $\left(y_{m}, \dot{\phi}_{m}\right)\left(y_{m+1}, \dot{\phi}_{m+1}\right)$ denote the corresponding values of $(y, \dot{\phi})$ when the light mass hits the floor right before and after the large mass hits the floor. Then, since $\dot{y}=O(\delta)$, we find that $y_{m+1}-y_{m}=O(\delta)$ and $\dot{\phi}_{m+1}-\dot{\phi}_{m}=O(\delta)$. As a consequence, $\left|\dot{\phi}_{m+1}\right| f\left(y_{m+1}\right)-\left|\dot{\phi}_{m}\right| f\left(y_{m}\right)=O(\delta)$

and the change in adiabatic invariant due to large mass hitting the floor is sufficiently small $\Delta I=O(\delta)$.

\section{Estimate of maximal number of collisions}

In this section, we estimate the maximal number of collisions of the dumbbell with the floor as a function of the mass ratios. As we have seen in Section 2.2, on $(Y-\phi)$ plane, the dumbbell reduces to a mass point that has unit velocity and elastic reflection. We use the classical billiard result which states that the number of collisions inside a straight wedge with the inner angle $\gamma$ is given by $N_{\gamma}=\lceil\pi / \gamma\rceil$, see e.g. [7].

\subsection{Boundaries on $Y-\phi$ plane}

First, we discuss the properties of the boundaries of the dumbbell system on $Y-\phi$ plane.

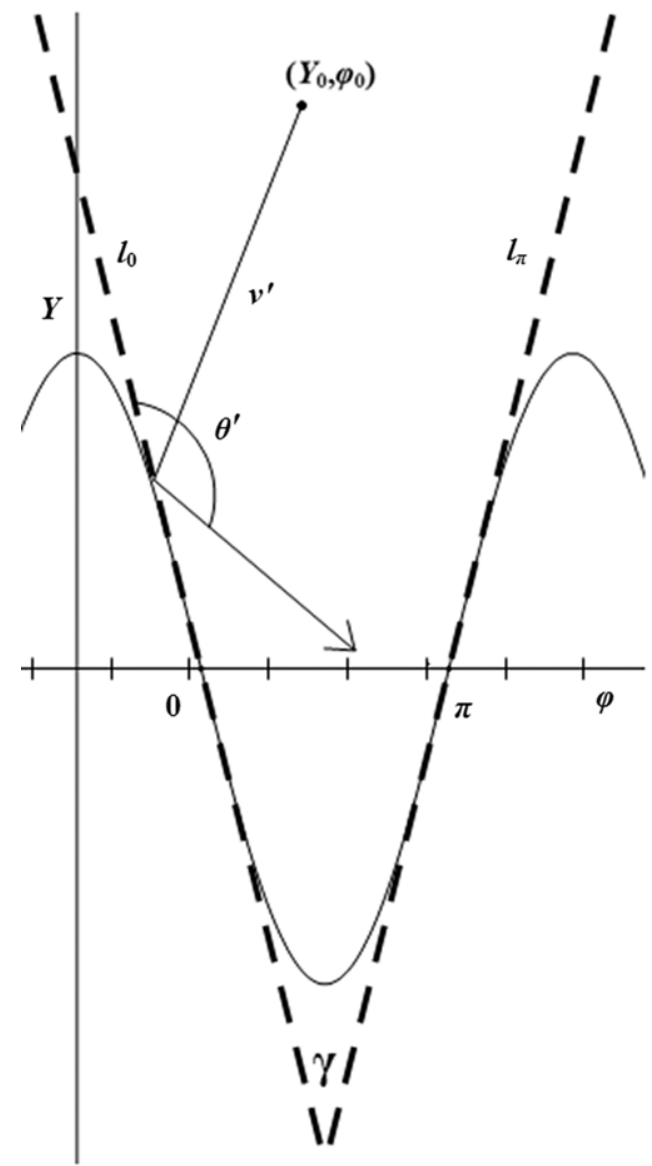

Fig. 3. Construction of the straight wedge and the hybrid wedge on $Y-\phi$ plane.

When $m_{1}=m_{2}$, we have the mass ratios $\beta_{1}=\beta_{2}=1 / 2$. Recall from $(2 c)$ that the boundaries are given by

$Y=\max \left\{-\sqrt{\beta_{2} / \beta_{1}} \sin \phi, \sqrt{\beta_{1} / \beta_{2}} \sin \phi\right\}=|\sin \phi|$

for $\phi \in[0,2 \pi]$.

Note that the angle between the two sine waves is $\gamma=\pi / 2$.

When $m_{1} \neq m_{2}$, it follows from (2c) that the boundaries consist of two sine curves with different heights. We will assume $m_{1}<m_{2}$, since the case $m_{2}<m_{1}$ is symmetric. It is easy to see that generically in the limit $m_{1} / m_{2} \rightarrow 0$ most of repeated collisions will occur between two peaks of (2a). In Section 4.2, we will find the upper bound for the number of collisions of the mass point to the boundaries. To start the proof, let us consider the straight wedge formed by the tangent lines to (2a) at $\phi=0$ and $\pi$. We call these tangent lines $\ell_{0}$ and $\ell_{\pi}$ respectively, and denote the angle of the straight wedge by $\gamma$, see Fig. 3 . Let us denote the wedge created by the union of the sine waves when $Y>0$ and the tangent lines $\ell_{0}$ and $\ell_{\pi}$ when $Y \leq 0$ as the hybrid wedge.

\subsection{The upper bound for the number of collisions}

We first introduce some notations. Denote the trajectory bouncing from the hybrid wedge by $v^{\prime}$, and let the approximating trajectory bouncing from the straight wedge by the double-prime symbols be $v^{\prime \prime}$. When $v^{\prime}$ or $v^{\prime \prime}$ is written with the subscript $i$, it denotes the segment of the corresponding trajectory between the $i$ th bounce and the $i+1$-st bounce. Let $\theta_{i}^{\prime}$ be the angle from the straight wedge to $v_{i}^{\prime}$, and $\theta_{i}^{\prime \prime}$ denote the angle from the straight wedge to $v_{i}^{\prime \prime}$ after the $i$ th collision. Define $\rho_{i}^{\prime}$ as the angle difference between the straight wedge and the hybrid wedge at ith collision of $v_{i}^{\prime}$. The trajectory will terminate when the sequence of angles terminates (due to the absence of the next bounce), or when there will be no 

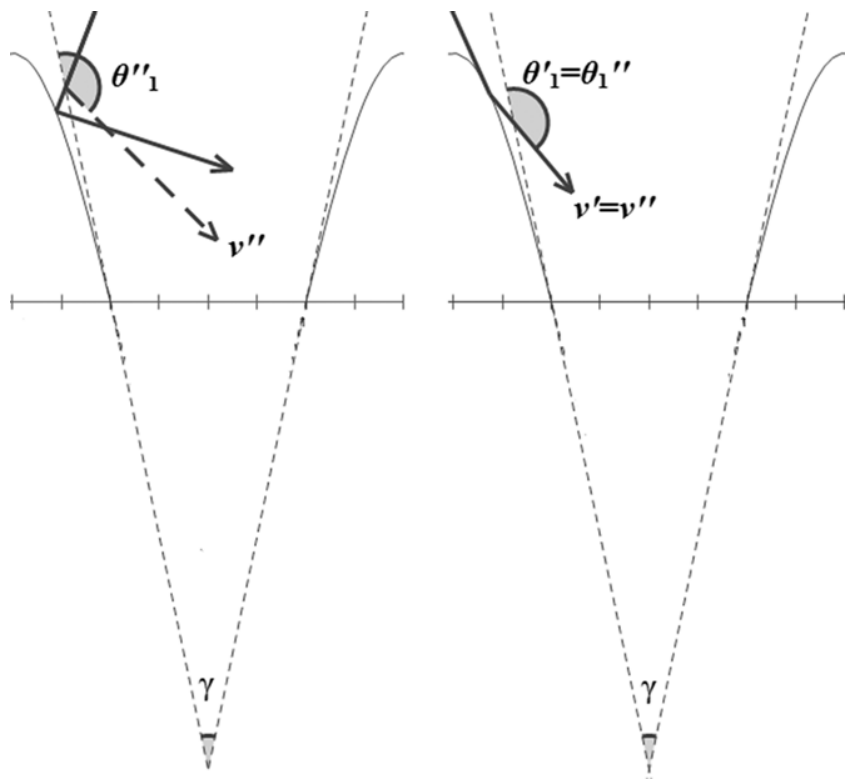

Fig. 4. Two different base cases for Lemma 4.1 .

more intersections with the straight wedge. This will happen when the angle of intersection, $\theta, \theta^{\prime}$ and $\theta^{\prime \prime}$, with the tangent line is less than or equal to $\gamma$.

Lemma 4.1. Consider the hybrid wedge and the straight wedge described above. The sequence of angles $\theta_{i}^{\prime \prime}, 1 \leq i$ will terminate after or at the same index as the sequence of angles $\bar{\theta}_{i}^{\prime}, 1 \leq i$.

Proof. Suppose that the initial segment $v_{0}^{\prime}$ (of the full trajectory) crosses the straight wedge before it hits the hybrid wedge, as shown on the left panel of Fig. 4. If the incoming angle is $\alpha$, then

$\theta_{1}^{\prime}=\pi-\alpha-2 \rho_{1}^{\prime}<\pi-\alpha=\theta_{1}^{\prime \prime}$.

When the initial segment $v_{0}^{\prime}$ hits the hybrid wedge before crossing the straight wedge, as shown on the right panel, then set $\theta_{1}^{\prime}=\theta_{1}^{\prime \prime}$. Now we can proceed by induction if $\theta_{i}^{\prime}>\gamma$ and $\theta_{i}^{\prime \prime}>\gamma$ and the sequence $\theta_{i}^{\prime}$ has not terminated.

$\theta_{i+1}^{\prime}=\theta_{i}^{\prime}-\gamma-2 \rho_{i+1}^{\prime}$

$\theta_{i+1}^{\prime \prime}=\theta_{i}^{\prime \prime}-\gamma$

which implies that $\theta_{i+1}^{\prime} \leq \theta_{i+1}^{\prime \prime}$.

Since $\theta_{i}^{\prime} \leq \theta_{i}^{\prime \prime}$, then $v^{\prime}$ will terminate at the same time or before $v^{\prime \prime}$.
Define the bridge as the smaller sine wave created by $Y=$ $-\sqrt{\beta_{2} / \beta_{1}} \sin \phi$ when $m_{1} \ll m_{2}$ from $\phi=0$ to $\phi=\pi$. The union of the bridge with the hybrid wedge will create the boundary as it is actually defined by the dumbbell dynamics.

Lemma 4.2. The presence of the bridge in the hybrid wedge will increase the number of collisions of the dumbbell by at most one from the number of collisions of the dumbbell to the hybrid wedge.

Proof. Consider the true trajectory (denoted by $v$ ) that "sees" the bridge. Recall the definition of angle $\theta_{i}^{\prime}$, which is the angle from the straight wedge to $v_{i}^{\prime}$. Similarly, we let $\theta_{i}$ be the angle to $v_{i}$. Before $v$ intersects the bridge, by Lemma 4.1 we have

$v_{i}=v_{i}^{\prime}, \quad \theta_{i}=\theta_{i}^{\prime}, \quad$ and $\theta_{i}=\theta_{i-1}-\gamma-2 \rho_{i}$.

Now define $\tau$ to be the angle measured from the horizontal line to the tangent line at the point where $v$ hits the bridge, see Fig. 5 . Note that $\tau$ takes a positive value if the dumbbell hits the left half of the bridge, and $\tau$ takes a negative value if $v$ hits the right half of the bridge. We express $\theta_{i+1}$ after the bounce from the bridge in terms of $\theta_{i}$. By this convention, the bounce from the bridge does not increase the index count but we will have to add +1 in the end.

Then, we have

$\theta_{i+1}=\pi-\theta_{i}-2 \tau-2 \rho_{i+1}$

$\theta_{i+1}^{\prime}=\theta_{i}^{\prime}-\gamma-2 \rho_{i+1}^{\prime}$.

We may assume that $v$ hits the bridge with non-positive velocity in $Y$. If the dumbbell hits the bridge with positive velocity in $Y$, it will continue to move in the positive $Y$ direction after reflection from the bridge. Then, we consider the reverse trajectory to bound the number of collisions. This allows us to restrict $\theta_{i}$. Moreover, $v_{i}$ naturally hits the upper part of hybrid wedge than $v_{i}^{\prime}$. We also assume that $v$ hits the left half of the bridge. Otherwise, we can reflect the orbit around the vertical line passing through the middle point of the bridge.

Utilizing the above arguments, we have the inequalities

$\frac{\pi+\gamma}{2}<\theta_{i}, \quad 0<\tau<\frac{\gamma}{2}, \quad$ and $\quad \rho_{i+1}^{\prime}<\rho_{i+1}$.

It is straightforward to verify that (8) and (9) imply $\theta_{i+1} \leq \theta_{i+1}^{\prime}$. From the $i+2$-nd bounce, if $\theta_{i}$ has not terminated, we can apply induction argument similar to the proof in Lemma 4.1. We have the base case

$\theta_{i+1} \leq \theta_{i+1}^{\prime}$, and $\rho_{i+1} \geq \rho_{i+1}^{\prime}$.

Note that $\rho$ 's indicate the relative position of a collision point in the hybrid wedge. That is, if $\rho_{i+1} \geq \rho_{i+1}^{\prime}$, then the starting point of $v_{i+1}$ is located at or above that of $v_{i}$. Since $\theta_{i+1} \leq \theta_{i+1}^{\prime}$ and $v_{i+1}$ starts

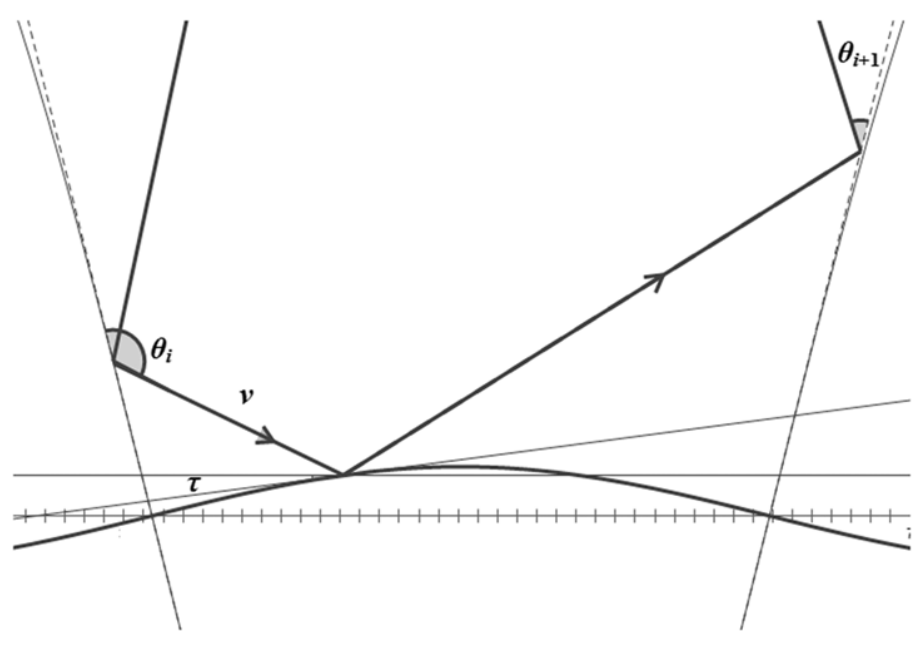

Fig. 5. The real trajectory $v$ that encounters the bridge. 
above $v_{i+1}^{\prime}$, we know $v_{i+2}$ will start on the hybrid wedge higher than $v_{i+2}^{\prime}$. This implies $\rho_{i+2} \leq \rho_{i+2}^{\prime}$. Then using the recursive relationship,

$\theta_{i+2}=\theta_{i+1}-\gamma-2 \rho_{i+2}$

$\theta_{i+2}^{\prime}=\theta_{i+1}^{\prime}-\gamma-2 \rho_{i+2}^{\prime}$,

we obtain $\theta_{i+2} \leq \theta_{i+2}^{\prime}$. By induction $\theta_{i} \leq \theta_{i}^{\prime}$ for all $i$. Taking into account the bounce on the bridge, we conclude that the number of bounces of $v$ will increase at most by one relative to that of $v^{\prime}$. Note that in most cases, the number of bounces of $v$ will be less than the number of bounces of $v^{\prime}$.

Now we are ready to prove the main theorem.

Theorem 4.3. The number of collisions of the dumbbell is bounded above by $N_{\gamma}=\lceil\pi / \gamma\rceil+1$, where $\gamma=\pi-2 \arctan \sqrt{\beta_{2} / \beta_{1}}$.

Proof. When $m_{1}=m_{2}$, as we have found in Section 4.1, the boundaries form identical hybrid wedges which intersect at $\pi / 2$. Using Lemma 4.1, we conclude that the upper bound for the number of collisions is $\lceil\pi / \gamma\rceil=2$, which is less than $N_{\gamma}=3$.

When $m_{1}<m_{2}$, we consider the true boundaries which consist of a hybrid wedge with the bridge. Using Lemmas 4.1 and 4.2 , we conclude that the maximal number of collisions to the true boundary is bounded above by $N_{\gamma}=\lceil\pi / \gamma\rceil+1$. Since $\gamma=\pi-2$ arctan $\sqrt{\beta_{2} / \beta_{1}}$, this completes the proof.

\section{Acknowledgments}

The authors acknowledge support from National Science Foundation grant DMS 08-38434 EMSW21-MCTP: "Research Experience for Graduate Students". YMB and VZ were also partially supported by NSF grant DMS-0807897. YMB and VB were also partially supported by ONR. The authors would also like to thank Mark Levi for a helpful discussion.

\section{References}

[1] R. Alleyne, Coin tossing through the ages, in: The Telegraph, 2009.

[2] J.B. Keller, The probability of heads, Amer. Math. Monthly 93 (1986) 191

[3] L. Mahadevan, E.H. Yong, Probability, physics, and a coin toss, Phys. Today (2011)

[4] P. Diaconis, S. Holmes, R. Montgomery, Dynamical Bias in the coin toss, SIAM Rev. 49 (2) (2007) 211-235.

[5] V.Z. Vulovic, R.E. Prange, Randomness of a true coin toss, Phys. Rev. A 33 (1986) $576-582$

[6] J.Sinai, Introduction to Ergodic Theory, in: Math. Notes, vol. 18, Princeton Univ. Press, Princeton, NJ, 1976.

[7] S. Tabachnikov, Geometry and Billiards, in: Geometry and Billiards, vol. 30, AMS, 2005.

[8] D. Burago, S. Ferleger, A. Kononenko, A geometric approach to semi-dispersing billiards (survey), Ergodic Theory Dynam. Systems 18 (1998) 303-319.

[9] D. Cowan, A billiard model for a gas of particles with rotation, Discrete Contin. Dyn. Syst. 22 (1\&2) (2008)

[10] L. Bobadilla, F. Martinez, E. Gobst, K. Gossman, S.M. LaValle, Controlling wild mobile robots using virtual gates and discrete transitions, in: American Control Conference, 2012. 